\title{
Exchange Rate Effects on Equity Prices: The Recent Case from Japan
}

\author{
Chikashi Tsuji ${ }^{1}$ \\ ${ }^{1}$ Faculty of Economics, Chuo University, 742-1 Higashinakano Hachioji-shi, Tokyo 192-0393 Japan \\ Correspondence: Chikashi Tsuji, Professor, Faculty of Economics, Chuo University, 742-1 Higashinakano \\ Hachioji-shi, Tokyo 192-0393 Japan. Tel: 81-42-674-2211. E-mail: mail_sec_low@minos.ocn.ne.jp
}

Received: September 27, 2015

Accepted: October 18, 2015

Online Published: October 19, 2015

doi:10.5430/bmr.v4n4p1

URL: http://dx.doi.org/10.5430/bmr.v4n4p1

\begin{abstract}
This paper investigates recent effects between the yen-US dollar exchange rate and Japanese stock prices. Applying bivariate Bayesian Vector Autoregressive (VAR) models, we obtain several clear findings. First, (1) our analyses using Bayesian VAR models find that, in our recent sample period, the first lags of the yen-dollar exchange rate are statistically significant in explaining four stock index prices of the Tokyo Stock Price Index (TOPIX), Tokyo Stock Exchange (TSE) Second Section stock index, TOPIX Large 70 stock index, and TOPIX Small stock index in Japan. Second, (2) our impulse response analyses find that, in our recent sample period, the above four stock index prices clearly respond to the shock in the yen-dollar exchange rate whilst the exchange rate little responds to the shock in these stock prices. Therefore, our results suggest that, in the recent years, past yen-dollar exchange rate series much more strongly affect Japanese stock prices whilst past Japanese stock price series have little effect on the yen-dollar exchange rate movements. Moreover, (3) our analyses of the time-varying correlation coefficients between the exchange rate changes and Japanese stock returns reveal that the contemporaneous correlations between them become much higher in the recent years.
\end{abstract}

Keywords: Bayesian VAR model, Yen-dollar exchange rate, Japanese stock price, Time-varying correlation

\section{Introduction}

In the recent years, as often reported in the media, Japanese stock prices are generally higher than before whilst the yen is depreciated than before. Then how are the time-series comovements of Japanese stock prices and the yen-US dollar exchange rate changes recently? Has the relationship between them changed in the recent years? Further, recently, do stock prices affect the exchange rate or does the exchange rate have an effect on stock prices? In order to derive the answers to these questions, by using the actual data, this paper tests the recent relationships between the yen-US dollar exchange rate and Japanese stock index prices. In empirical analyses, we employ Bayesian Vector Autoregressive (VAR) models in this paper.

This study provides the following interesting and clear findings. First, (1) our research using Bayesian VAR models clarifies that recently, the first lags of the yen-dollar exchange rate statistically significantly explain the movements of four Japanese stock index prices; namely, the Tokyo Stock Price Index (TOPIX), Tokyo Stock Exchange (TSE) Second Section stock index, TOPIX Large 70 stock index, and TOPIX Small stock index in Japan. Next, (2) our impulse response analyses further reveal that, recently, the above four Japanese stock index prices clearly react to the shock to the yen-dollar exchange rate, however, the exchange rate little reacts to the shock to the stock prices. Thus these results indicate that, in the recent years, the past yen-dollar exchange rate much more strongly influences Japanese stock prices; while past Japanese stock prices have little influence on the yen-dollar exchange rate. Further, (3) our analyses of the time-varying correlation coefficients of the exchange rate and Japanese stock returns find that, in the recent years, much higher contemporaneous correlations between them are observed. After this introduction, we review existing studies in Section 2; our data are explained in Section 3; our methodology is described in Section 4; our results are explained in Section 5; and in Section 6, our conclusions are presented.

\section{Literature Review}

Focusing on the recent studies, this section conducts related literature review. First, Liang, Lin, and Hsu (2013) revisited the relations between stock markets and currency markets of the Association of South-East Asian Nations (ASEAN) countries with the panel Granger causality methodologies. They concluded that their results were supportive for the 'stock-oriented' hypothesis of exchange rates by Branson (1983) and Frankel (1983), which 
suggested that exchange rates affect stock prices negatively through capital mobility. Ciner, Gurdgiev, and Lucey (2013) examined the return relationships between major asset classes using the US and UK data. They derived the evidence that both in the US and UK, we can regard gold as a safe haven against exchange rates. In addition, Vithessonthi and Tongurai (2013) investigated the effects of the imposition of Thailand's unremunerated reserve requirement on capital inflows in 2006-2007 on exchange rate volatility. They showed that during the period of the imposition of the unremunerated reserve requirement in Thailand, the exchange rate volatility of the Thai baht against the US dollar, the euro, the British pound, and the Japanese yen seemed to be larger.

Further, Moore and Wang (2014) investigated the determinants of the time-varying correlations between real exchange rates and stock returns for four developed markets and six Asian emerging markets. Andrieş, Ihnatov, and Tiwari (2014) attempted to identify the comovement patterns of stock price, exchange rate, and interest rate in India from July 1997 to December 2010 by applying some cross-wavelet methodologies. Moreover, a very recent study by Ho and Huang (2015) investigated the nonlinear connections between stock price indices and exchange rates of China, India, Russia, and Brazil. Abouwafia and Chambers (2015) explored the linkages among monetary policy, stock prices, and exchange rates in the Middle East region. Gelman et al. (2015) examined the associations between capital flows and real exchange rates. Furthermore, Ca'Zorzi, Kocięcki, and Rubaszek (2015) implemented a Bayesian forecasting of real exchange rates with a Dornbusch prior. Petrevski et al. (2015) examined the transmission of various foreign shocks to South Eastern European economies by using a Bayesian VAR approach. As the above review of recent studies shows, we understand that the time-series linkage of the yen-dollar exchange rate changes and Japanese stock price evolution in the recent period has been little analyzed in existing literature.

\section{Data}

In this study, we use four Japanese equity index and one exchange rate data. Specifically, EXLR means the percentage log changes as to the yen-US dollar exchange rate, TXLR denotes the percentage log return of the TOPIX, TSE2LR denotes the percentage log return of the TSE Second Section stock index, TL70LR means that of the TOPIX Large 70 stock index, and TSMLR denotes that of the TOPIX Small stock index in Japan.

We further use the level variables related to the above five variables. Namely, EX represents the level variable of the yen-dollar exchange rate; TX denotes the level variable of the TOPIX; TSE2 represents the level variable of the TSE Second Section stock index; TL70 means that of the TOPIX Large 70 stock index; and TSM denotes that of the TOPIX Small stock index in Japan. In addition, this paper analyzes two sample periods: the first is (1) the period from April 1, 2010 to November 13, 2012 and the second is (2) that from December 26, 2012 to August 18, 2015. This study uses data from the QUICK Corp.

Table 1 shows the descriptive statistics as to the above five log return (change) variables. This table indicates the following characteristics of the return data. First, (1) the mean values of the five variables are all negative for our first sample period from April 1, 2010 to November 13, 2012 while they are all positive for our second sample period from December 26, 2012 to August 18, 2015. Further, (2) the kurtosis values of the five variables are clearly higher for our first period than those for our second period. Third, (3) for our first and second sample periods, the skewness values of the five variables are all slightly negative except for the exchange rate change variable, EXLR.

\section{Methodology}

This paper employs the following analyzing methodology and models. For examining the dynamic relations of the above four kinds of Japanese stock index prices and the yen-dollar exchange rate, (1) we first calculate the time-varying correlation coefficients by taking 20-days windows and compare them in our two sample periods explained above. (2) Second, we estimate four sorts of bivariate Bayesian VAR models by using the above level variables and investigate the impulse response functions derived from those models. More concretely, the econometric models we estimate in this study are the bivariate Bayesian VAR(4) models of (1) EX and TX; (2) EX and TSE2; (3) EX and TL70; and (4) EX and TSM. As the following equations (1) and (2), all our VAR(4) models can be summarized as two equations:

$$
\begin{aligned}
& e x_{t}=\tau_{1}+\sum_{h=1}^{p} \zeta_{1, h} e x_{t-h}+\sum_{j=1}^{q} \psi_{1, j} s p_{t-j}+\kappa_{1, t}, \\
& s p_{t}=\tau_{2}+\sum_{r=1}^{p} \zeta_{2, r} e x_{t-r}+\sum_{s=1}^{q} \psi_{2, s} s p_{t-s}+\kappa_{2, t} .
\end{aligned}
$$

In the above VARs, we always specify the lag orders as $p=4$ and $q=4$ to conduct consistent tests in our study. In the above models, $e x$ means the yen-dollar exchange rate level and $s p$ is one of the four Japanese equity index prices: the TOPIX, TSE Second Section stock index, TOPIX Large 70 stock index, or TOPIX Small stock index in Japan. 
Table 1. Descriptive statistics of the Japanese stock index returns and the yen-dollar exchange rate changes: For the period from April 1, 2010 to November 13, 2012 and December 26, 2012 to August 18, 2015

\begin{tabular}{|c|c|c|c|c|}
\hline \multicolumn{5}{|c|}{ Panel A. Statistics for the period from April 1, 2010 to November 13, 2012} \\
\hline & EXLR & TXLR & & TSE2LR \\
\hline Mean & -0.0251 & -0.0469 & & -0.0010 \\
\hline Median & -0.0297 & -0.0320 & & 0.0600 \\
\hline Maximum & 3.8351 & 6.4275 & & 5.7307 \\
\hline Minimum & -2.1419 & -9.9519 & & -11.5827 \\
\hline Standard deviation & 0.5693 & 1.2168 & & 0.9002 \\
\hline Skewness & 1.1281 & -0.9851 & & -4.6266 \\
\hline Kurtosis & 9.9756 & 12.4999 & & 72.1236 \\
\hline \multirow[t]{2}{*}{ Observations } & 647 & 647 & & 647 \\
\hline & TL70LR & & \multicolumn{2}{|l|}{ TSMLR } \\
\hline Mean & -0.0508 & & \multicolumn{2}{|l|}{-0.0182} \\
\hline Median & -0.0465 & & \multicolumn{2}{|l|}{0.0431} \\
\hline Maximum & 7.3691 & & \multicolumn{2}{|l|}{7.1139} \\
\hline Minimum & -9.6662 & & \multicolumn{2}{|l|}{-13.1830} \\
\hline Standard deviation & 1.3363 & & \multicolumn{2}{|l|}{1.2727} \\
\hline Skewness & -0.5960 & & \multicolumn{2}{|l|}{-2.1904} \\
\hline Kurtosis & 9.4533 & & \multicolumn{2}{|l|}{27.7828} \\
\hline Observations & 647 & & \multicolumn{2}{|l|}{647} \\
\hline
\end{tabular}

Panel B. Statistics for the period from December 26, 2012 to August 18, 2015

\begin{tabular}{|c|c|c|c|c|}
\hline & EXLR & TXLR & & TSE2LR \\
\hline Mean & 0.0590 & 0.1068 & & 0.1123 \\
\hline Median & 0.0554 & 0.1320 & & 0.1570 \\
\hline Maximum & 3.1691 & 5.0746 & & 4.0984 \\
\hline Minimum & -2.7370 & -7.1196 & & -4.4570 \\
\hline Standard deviation & 0.6142 & 1.2621 & & 0.8547 \\
\hline Skewness & 0.2390 & -0.4870 & & -0.8902 \\
\hline Kurtosis & 6.5219 & 5.7782 & & 7.2138 \\
\hline \multirow[t]{2}{*}{ Observations } & 647 & 647 & & 647 \\
\hline & TL70LR & & TSMLR & \\
\hline Mean & 0.1109 & & 0.1023 & \\
\hline Median & 0.1397 & & 0.1870 & \\
\hline Maximum & 4.4878 & & 5.7778 & \\
\hline Minimum & -7.6730 & & -7.0532 & \\
\hline Standard deviation & 1.3066 & & 1.1978 & \\
\hline Skewness & -0.5802 & & -0.6440 & \\
\hline Kurtosis & 5.9119 & & 7.1629 & \\
\hline Observations & 647 & & 647 & \\
\hline
\end{tabular}

Notes: Descriptive statistics for the analyzed stock return and exchange rate change variables are shown in this table. The statistics are shown for two sample periods. Further, EXLR means the percentage log changes of the yen-dollar exchange rate series, TXLR denotes the percentage log return of the TOPIX, TSE2LR denotes the percentage log return of the TSE Second Section stock index, TL70LR means that of the TOPIX Large 70 stock index, and TSMLR denotes that of the TOPIX Small stock index in Japan. 
Panel A. EXLR and TXLR

April 1, 2010 to November 13, 2012

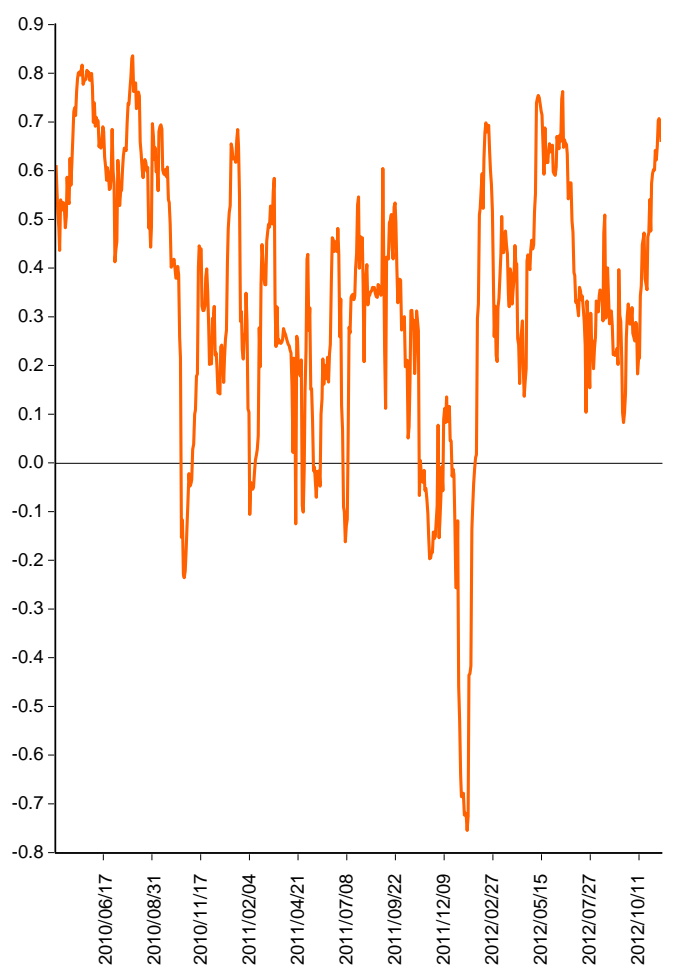

Panel B. EXLR and TSE2LR

April 1, 2010 to November 13, 2012

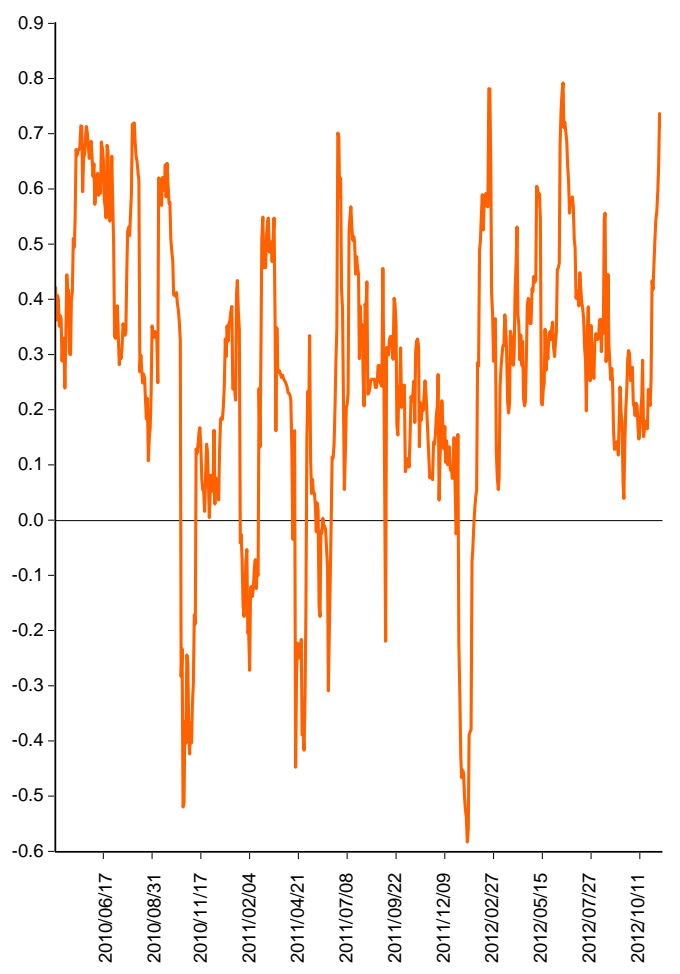

December 26, 2012 to August 18, 2015

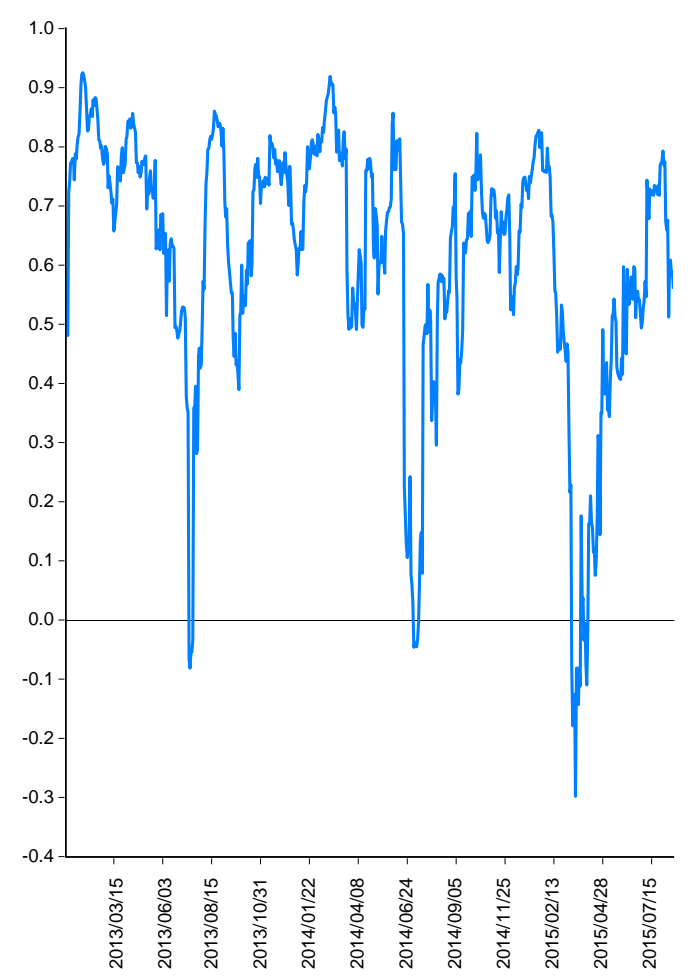

December 26, 2012 to August 18, 2015

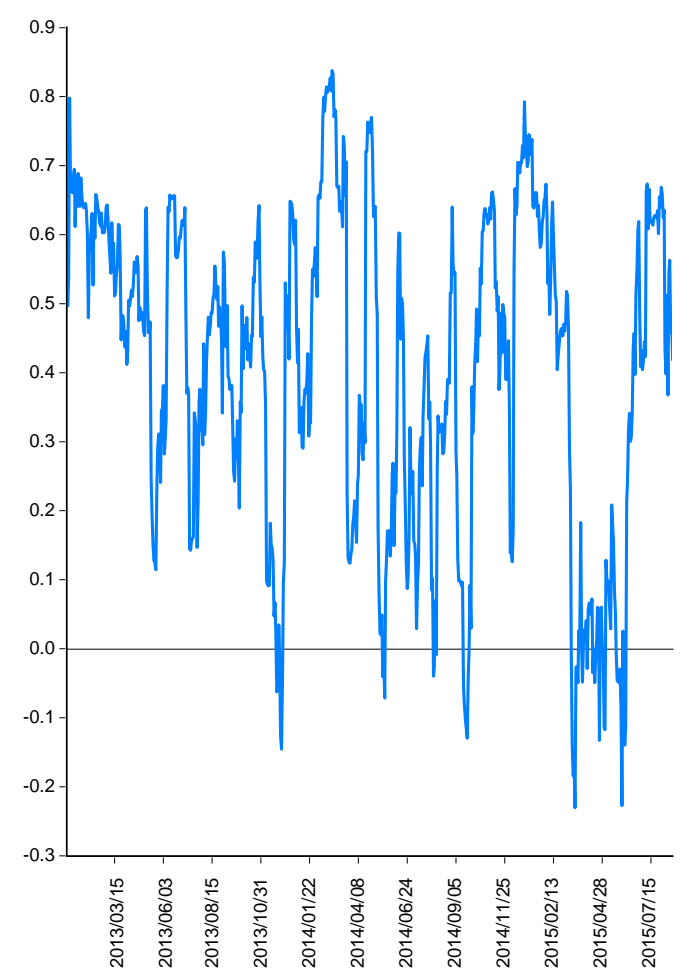


Panel C. EXLR and TL70LR

April 1, 2010 to November 13, 2012

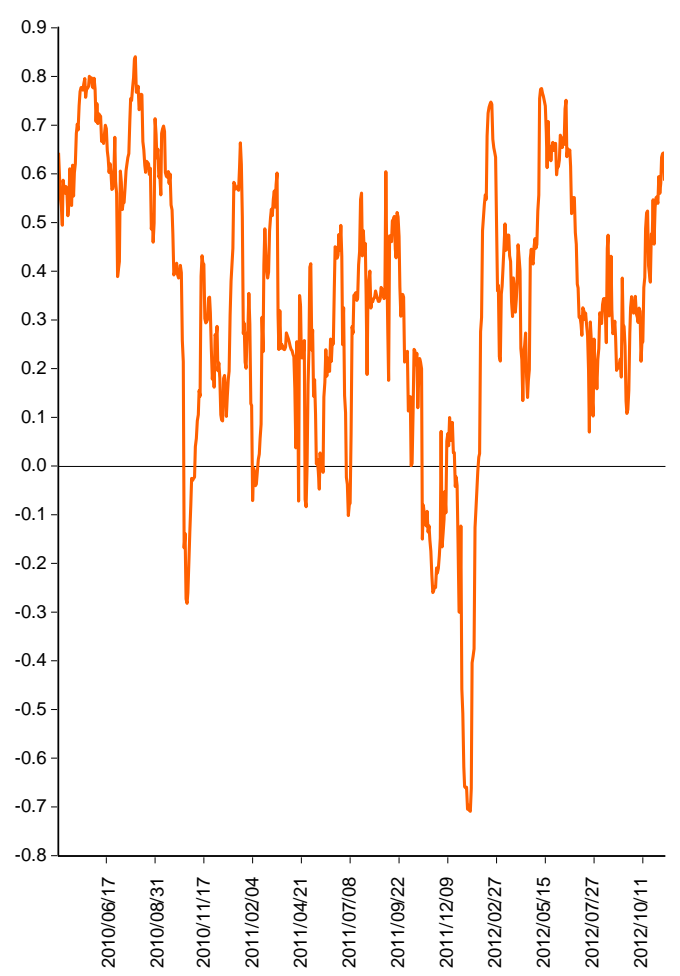

Panel D. EXLR and TSMLR

April 1, 2010 to November 13, 2012

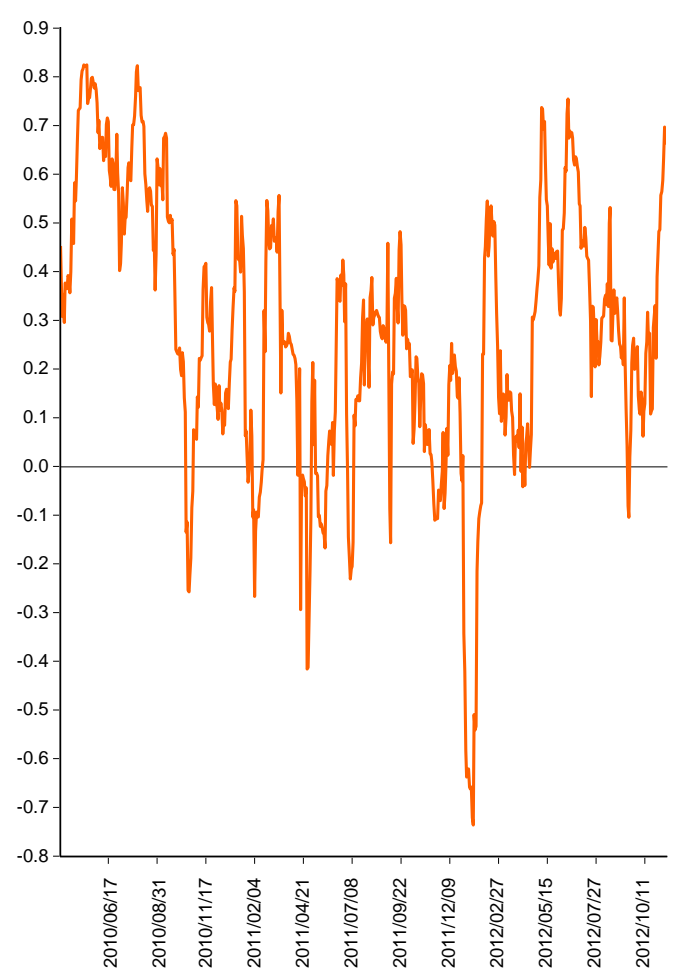

December 26, 2012 to August 18, 2015

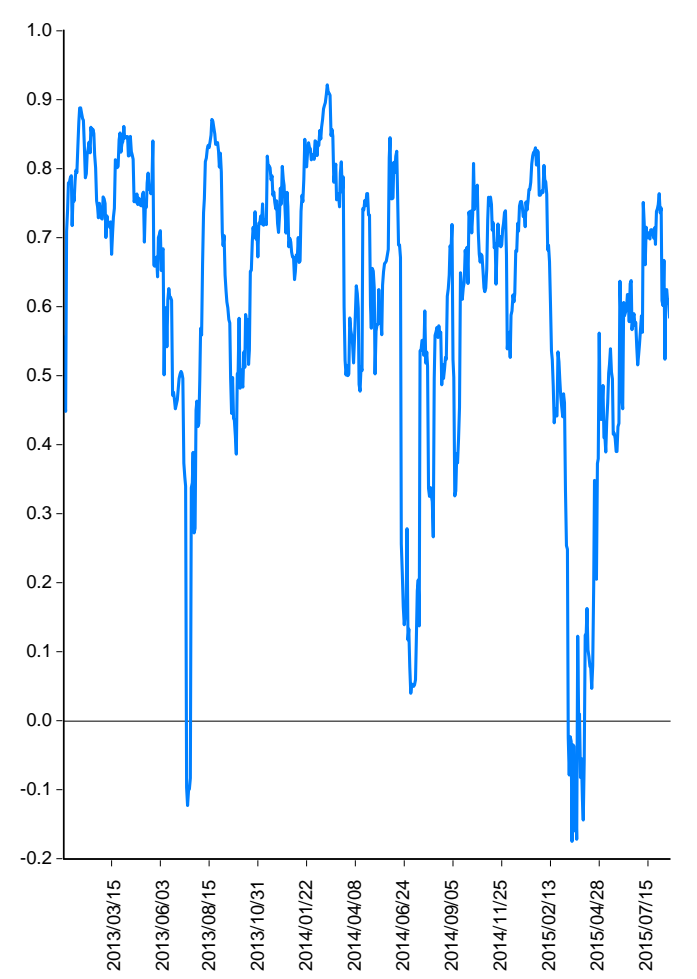

December 26, 2012 to August 18, 2015

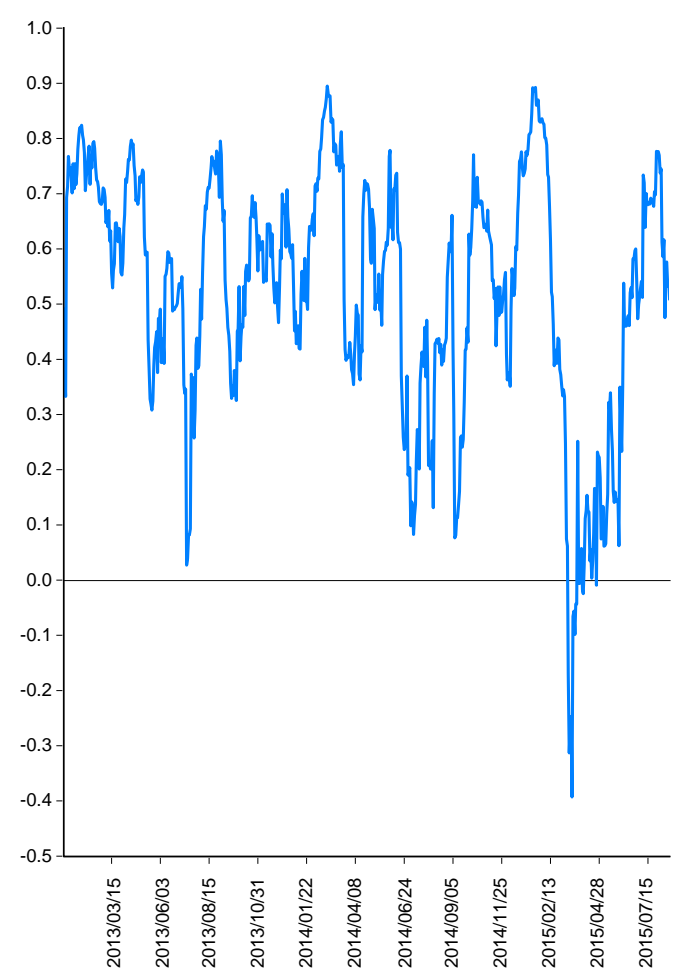

Figure 1. Time-series evolution of the time-varying correlations between four kinds of Japanese stock index returns and the yen-dollar exchange rate changes 
Table 2. Estimation results of the bivariate Bayesian VAR models: For the period from April 1, 2010 to November 13, 2012 and from December 26, 2012 to August 18, 2015

\begin{tabular}{|c|c|c|c|c|c|}
\hline \multicolumn{6}{|c|}{ Panel A. Estimation results for April 1, 2010 to November 13, 2012} \\
\hline \multirow[t]{2}{*}{ Model 1} & \multirow{2}{*}{\multicolumn{3}{|c|}{ Model 2}} & \multirow[b]{2}{*}{ EX } & \multirow[b]{2}{*}{ TSE2 } \\
\hline & & & & & \\
\hline $\mathrm{EX}(-1)$ & $0.7918 * * *$ & 0.6728 & $\mathrm{EX}(-1)$ & $0.7985^{* * *}$ & 0.5128 \\
\hline$t$-value & 24.1340 & 0.9592 & $t$-value & 24.8054 & 0.4054 \\
\hline$p$-value & 0.0000 & 0.3378 & $p$-value & 0.0000 & 0.6853 \\
\hline $\mathrm{EX}(-2)$ & $0.1285^{* * *}$ & $-1.1969 *$ & $\mathrm{EX}(-2)$ & $0.1298 * * *$ & -1.6333 \\
\hline$t$-value & 3.8360 & -1.6753 & $t$-value & 3.8991 & -1.2521 \\
\hline$p$-value & 0.0001 & 0.0944 & $p$-value & 0.0001 & 0.2110 \\
\hline $\mathrm{EX}(-3)$ & $0.0484 *$ & 0.3830 & $\mathrm{EX}(-3)$ & $0.0470^{*}$ & 0.4967 \\
\hline$t$-value & 1.8866 & 0.7014 & $t$-value & 1.8457 & 0.4978 \\
\hline$p$-value & 0.0597 & 0.4833 & $p$-value & 0.0654 & 0.6188 \\
\hline $\mathrm{EX}(-4)$ & 0.0118 & 0.2004 & $\mathrm{EX}(-4)$ & 0.0107 & 0.5419 \\
\hline$t$-value & 0.5996 & 0.4762 & $t$-value & 0.5437 & 0.7033 \\
\hline$p$-value & 0.5490 & 0.6341 & $p$-value & 0.5869 & 0.4821 \\
\hline $\mathrm{TX}(-1)$ & 0.0020 & $0.9203 * * *$ & TSE2(-1) & 0.0008 & $1.1010^{* * *}$ \\
\hline$t$-value & 1.3550 & 28.8704 & $t$-value & 1.0346 & 37.5052 \\
\hline$p$-value & 0.1759 & 0.0000 & $p$-value & 0.3012 & 0.0000 \\
\hline $\mathrm{TX}(-2)$ & 0.0017 & 0.0524 & TSE2(-2) & 0.0012 & $-0.0896^{* *}$ \\
\hline$t$-value & 1.0807 & 1.5183 & $t$-value & 1.3255 & -2.5526 \\
\hline$p$-value & 0.2803 & 0.1294 & $p$-value & 0.1855 & 0.0109 \\
\hline $\mathrm{TX}(-3)$ & -0.0016 & 0.0182 & TSE2(-3) & $-0.0012 *$ & -0.0228 \\
\hline$t$-value & -1.3273 & 0.7098 & $t$-value & -1.8630 & -0.8941 \\
\hline$p$-value & 0.1849 & 0.4781 & $p$-value & 0.0629 & 0.3716 \\
\hline $\mathrm{TX}(-4)$ & $-0.0017 *$ & -0.0091 & TSE2(-4) & -0.0008 & -0.0111 \\
\hline$t$-value & -1.8620 & -0.4702 & $t$-value & -1.6303 & -0.6037 \\
\hline$p$-value & 0.0631 & 0.6384 & $p$-value & 0.1035 & 0.5462 \\
\hline Constant term & $1.1738 * * *$ & 9.6197 & Constant term & $1.1392 * *$ & $56.5466 * * *$ \\
\hline$t$-value & 3.2761 & 1.2516 & $t$-value & 2.3528 & 2.9621 \\
\hline$p$-value & 0.0011 & 0.2112 & $p$-value & 0.0189 & 0.0032 \\
\hline $\operatorname{Adj.} R^{2}$ & 0.9882 & 0.9800 & Adj. $R^{2}$ & 0.9883 & 0.9713 \\
\hline \multirow[t]{2}{*}{ Model 3} & & & Model 4 & & \\
\hline & EX & TL70 & & EX & TSM \\
\hline $\mathrm{EX}(-1)$ & $0.7947 * * *$ & 0.7182 & $\mathrm{EX}(-1)$ & 0.7978 *** & 0.3118 \\
\hline$t$-value & 24.2799 & 0.9672 & $t$-value & 24.6362 & 0.4113 \\
\hline$p$-value & 0.0000 & 0.3338 & $p$-value & 0.0000 & 0.6810 \\
\hline $\mathrm{EX}(-2)$ & $0.1282 * * *$ & $-1.3119 *$ & $\mathrm{EX}(-2)$ & $0.1289 * * *$ & -1.0251 \\
\hline$t$-value & 3.8285 & -1.7308 & $t$-value & 3.8633 & -1.3151 \\
\hline$p$-value & 0.0001 & 0.0840 & $p$-value & 0.0001 & 0.1890 \\
\hline $\mathrm{EX}(-3)$ & $0.0478^{*}$ & 0.4173 & $\mathrm{EX}(-3)$ & $0.0473 *$ & 0.4251 \\
\hline$t$-value & 1.8652 & 0.7203 & $t$-value & 1.8544 & 0.7138 \\
\hline$p$-value & 0.0626 & 0.4716 & $p$-value & 0.0641 & 0.4756 \\
\hline $\mathrm{EX}(-4)$ & 0.0115 & 0.1868 & $\mathrm{EX}(-4)$ & 0.0111 & 0.3168 \\
\hline$t$-value & 0.5820 & 0.4183 & $t$-value & 0.5614 & 0.6896 \\
\hline$p$-value & 0.5608 & 0.6759 & $p$-value & 0.5747 & 0.4907 \\
\hline
\end{tabular}




\begin{tabular}{|c|c|c|c|c|c|}
\hline \multicolumn{2}{|c|}{ www.sciedupress.com/bmr } & \multicolumn{2}{|c|}{ Business and Management Research } & & Vol. 4, No. 4; 2015 \\
\hline TL70(-1) & 0.0016 & $0.9083 * * *$ & $\mathrm{TSM}(-1)$ & 0.0014 & $0.9791 * * *$ \\
\hline$t$-value & 1.1734 & 28.4603 & $t$-value & 1.0381 & 31.5763 \\
\hline$p$-value & 0.2411 & 0.0000 & $p$-value & 0.2996 & 0.0000 \\
\hline $\operatorname{TL70}(-2)$ & 0.0017 & $0.0611 *$ & $\operatorname{TSM}(-2)$ & 0.0020 & -0.0034 \\
\hline$t$-value & 1.1533 & 1.7771 & $t$-value & 1.3666 & -0.0988 \\
\hline$p$-value & 0.2492 & 0.0760 & $p$-value & 0.1722 & 0.9213 \\
\hline $\operatorname{TL70}(-3)$ & -0.0015 & 0.0251 & $\operatorname{TSM}(-3)$ & -0.0016 & -0.0108 \\
\hline$t$-value & -1.3157 & 0.9794 & $t$-value & -1.5049 & -0.4235 \\
\hline$p$-value & 0.1887 & 0.3278 & $p$-value & 0.1328 & 0.6721 \\
\hline $\operatorname{TL} 70(-4)$ & $-0.0016^{*}$ & -0.0082 & $\operatorname{TSM}(-4)$ & $-0.0015^{*}$ & -0.0042 \\
\hline$t$-value & -1.8469 & -0.4186 & $t$-value & -1.8310 & -0.2190 \\
\hline$p$-value & 0.0652 & 0.6756 & $p$-value & 0.0676 & 0.8267 \\
\hline Constant term & $1.1665^{* * * *}$ & 9.4807 & Constant term & $0.9402 * *$ & $32.6558 * * *$ \\
\hline$t$-value & 3.2525 & 1.1612 & $t$-value & 2.2622 & 3.3447 \\
\hline$p$-value & 0.0012 & 0.2460 & $p$-value & 0.0240 & 0.0009 \\
\hline $\operatorname{Adj} . R^{2}$ & 0.9882 & 0.9814 & $\operatorname{Adj.} R^{2}$ & 0.9882 & 0.9460 \\
\hline
\end{tabular}

Panel B. Estimation results for December 26, 2012 to August 18, 2015

\begin{tabular}{|c|c|c|c|c|c|}
\hline \multirow[t]{2}{*}{ Model 1} & \multicolumn{4}{|c|}{ Model 2} & \multirow[b]{2}{*}{ TSE2 } \\
\hline & $\mathrm{EX}$ & $\mathrm{TX}$ & & $\mathrm{EX}$ & \\
\hline $\mathrm{EX}(-1)$ & $0.8543 * * *$ & $2.4049 * * *$ & $\mathrm{EX}(-1)$ & $0.8721 * * *$ & $3.1354 * *$ \\
\hline$t$-value & 24.6626 & 2.8675 & $t$-value & 26.6532 & 2.0054 \\
\hline$p$-value & 0.0000 & 0.0043 & $p$-value & 0.0000 & 0.0453 \\
\hline $\mathrm{EX}(-2)$ & $0.0951 * * *$ & $-1.5124^{*}$ & $\mathrm{EX}(-2)$ & $0.0859 * *$ & -1.6423 \\
\hline$t$-value & 2.7198 & -1.7908 & $t$-value & 2.5013 & -1.0036 \\
\hline$p$-value & 0.0067 & 0.0738 & $p$-value & 0.0126 & 0.3160 \\
\hline $\mathrm{EX}(-3)$ & 0.0289 & -0.1125 & $\mathrm{EX}(-3)$ & 0.0151 & -0.5541 \\
\hline$t$-value & 1.1024 & -0.1777 & $t$-value & 0.5865 & -0.4511 \\
\hline$p$-value & 0.2707 & 0.8590 & $p$-value & 0.5577 & 0.6521 \\
\hline $\mathrm{EX}(-4)$ & 0.0171 & -0.2584 & $\mathrm{EX}(-4)$ & 0.0072 & -0.1063 \\
\hline$t$-value & 0.8582 & -0.5363 & $t$-value & 0.3666 & -0.1135 \\
\hline$p$-value & 0.3911 & 0.5919 & $p$-value & 0.7141 & 0.9097 \\
\hline $\mathrm{TX}(-1)$ & $0.0034 * *$ & $0.8718 * * *$ & $\operatorname{TSE} 2(-1)$ & 0.0011 & $0.9900 * * *$ \\
\hline$t$-value & 2.4006 & 25.1666 & $t$-value & 1.6315 & 31.2542 \\
\hline$p$-value & 0.0167 & 0.0000 & $p$-value & 0.1033 & 0.0000 \\
\hline $\mathrm{TX}(-2)$ & -0.0014 & $0.0936 * * *$ & TSE2(-2) & -0.0007 & -0.0067 \\
\hline$t$-value & -0.9374 & 2.6510 & $t$-value & -1.0029 & -0.1909 \\
\hline$p$-value & 0.3489 & 0.0082 & $p$-value & 0.3163 & 0.8487 \\
\hline $\mathrm{TX}(-3)$ & -0.0018 & -0.0070 & TSE2(-3) & -0.0004 & 0.0013 \\
\hline$t$-value & -1.6368 & -0.2654 & $t$-value & -0.6865 & 0.0509 \\
\hline$p$-value & 0.1022 & 0.7908 & $p$-value & 0.4926 & 0.9594 \\
\hline $\mathrm{TX}(-4)$ & -0.0002 & 0.0122 & TSE2(-4) & 0.0003 & 0.0020 \\
\hline$t$-value & -0.2440 & 0.6136 & $t$-value & 0.7302 & 0.1026 \\
\hline$p$-value & 0.8073 & 0.5397 & $p$-value & 0.4655 & 0.9183 \\
\hline Constant term & 0.4013 & $-16.4774 *$ & Constant term & $1.1506^{* *}$ & -33.8077 \\
\hline$t$-value & 0.9847 & -1.6644 & $t$-value & 2.2470 & -1.3771 \\
\hline$p$-value & 0.3251 & 0.0965 & $p$-value & 0.0250 & 0.1690 \\
\hline $\operatorname{Adj} . R^{2}$ & 0.9960 & 0.9938 & $A d j . R^{2}$ & 0.9960 & 0.9979 \\
\hline Published by Sci & Press & 7 & & ISSN 1927-6001 & E-ISSN 1927-601X \\
\hline
\end{tabular}




\begin{tabular}{|c|c|c|c|c|c|}
\hline \multirow[t]{2}{*}{ Model 3} & \multicolumn{5}{|c|}{ Model 4} \\
\hline & EX & TL70 & & EX & TSM \\
\hline $\mathrm{EX}(-1)$ & $0.8510 * * *$ & $2.3291 * * *$ & $\mathrm{EX}(-1)$ & $0.8647 * * *$ & $2.1588 * *$ \\
\hline$t$-value & 24.4914 & 2.8356 & $t$-value & 25.7645 & 2.4463 \\
\hline$p$-value & 0.0000 & 0.0047 & $p$-value & 0.0000 & 0.0147 \\
\hline $\mathrm{EX}(-2)$ & $0.0947 * * *$ & $-1.4402 *$ & $\mathrm{EX}(-2)$ & $0.0900 * * *$ & -1.4257 \\
\hline$t$-value & 2.7070 & -1.7458 & $t$-value & 2.5989 & -1.5697 \\
\hline$p$-value & 0.0070 & 0.0813 & $p$-value & 0.0096 & 0.1170 \\
\hline $\mathrm{EX}(-3)$ & 0.0289 & -0.0557 & $\mathrm{EX}(-3)$ & 0.0233 & -0.1264 \\
\hline$t$-value & 1.1019 & -0.0901 & $t$-value & 0.8947 & -0.1856 \\
\hline$p$-value & 0.2709 & 0.9283 & $p$-value & 0.3713 & 0.8528 \\
\hline $\mathrm{EX}(-4)$ & 0.0172 & -0.2562 & $\mathrm{EX}(-4)$ & 0.0136 & -0.1102 \\
\hline$t$-value & 0.8626 & -0.5446 & $t$-value & 0.6903 & -0.2129 \\
\hline$p$-value & 0.3887 & 0.5863 & $p$-value & 0.4903 & 0.8315 \\
\hline $\operatorname{TL} 70(-1)$ & $0.0037 * *$ & $0.8758 * * *$ & $\operatorname{TSM}(-1)$ & $0.0026^{* *}$ & $0.8826 * * *$ \\
\hline$t$-value & 2.5389 & 25.2488 & $t$-value & 2.0621 & 26.2611 \\
\hline$p$-value & 0.0114 & 0.0000 & $p$-value & 0.0396 & 0.0000 \\
\hline TL70(-2) & -0.0014 & $0.0929 * * *$ & $\operatorname{TSM}(-2)$ & -0.0010 & $0.0764 * *$ \\
\hline$t$-value & -0.9260 & 2.6274 & $t$-value & -0.7598 & 2.1870 \\
\hline$p$-value & 0.3548 & 0.0088 & $p$-value & 0.4477 & 0.0291 \\
\hline $\operatorname{TL} 70(-3)$ & -0.0018 & -0.0079 & $\operatorname{TSM}(-3)$ & -0.0013 & 0.0051 \\
\hline$t$-value & -1.6209 & -0.2993 & $t$-value & -1.3247 & 0.1968 \\
\hline$p$-value & 0.1055 & 0.7648 & $p$-value & 0.1858 & 0.8441 \\
\hline TL70(-4) & -0.0003 & 0.0087 & $\operatorname{TSM}(-4)$ & $-3.1 \mathrm{E}-05$ & 0.0100 \\
\hline$t$-value & -0.3166 & 0.4369 & $t$-value & -0.0413 & 0.5052 \\
\hline$p$-value & 0.7516 & 0.6623 & $p$-value & 0.9671 & 0.6136 \\
\hline Constant term & 0.5823 & $-23.2006 * *$ & Constant term & 0.5182 & -13.1442 \\
\hline$t$-value & 1.1712 & -1.9676 & $t$-value & 1.4124 & -1.3580 \\
\hline$p$-value & 0.2419 & 0.0495 & $p$-value & 0.1583 & 0.1749 \\
\hline $\operatorname{Adj.} R^{2}$ & 0.9960 & 0.9945 & $\operatorname{Adj.R^{2}}$ & 0.9960 & 0.9937 \\
\hline
\end{tabular}

Notes: This table displays the estimation results of our four VAR(4) models for the yen-dollar exchange rate and four kinds of stock index prices in Japan. In this table, EX means the level variable of the yen-dollar exchange rate, TX denotes the level variable of the TOPIX, TSE2 denotes the level variable of the TSE Second Section stock index, TL70 means that of the TOPIX Large 70 stock index, and TSM denotes that of the TOPIX Small stock index in Japan. Moreover, $A d j . R^{2}$ means the adjusted- $R$ squared value and $* * *, * *$, and $*$ indicate the statistical significance of the coefficients at the 1,5 , and $10 \%$ levels, respectively.

Panel A. Accumulated Response of TX to EX

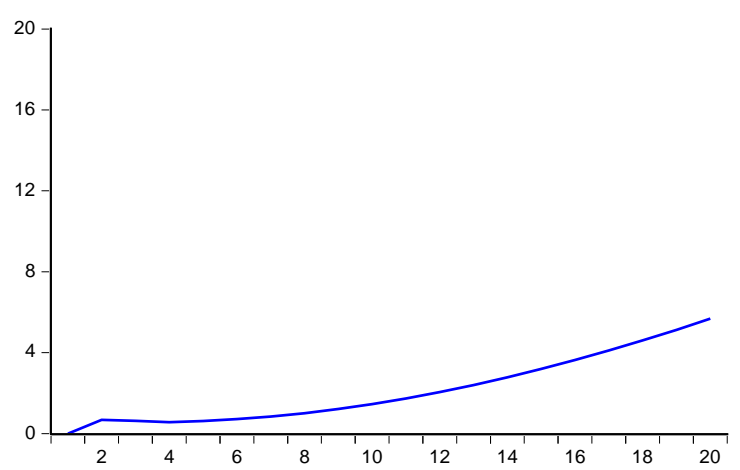

Panel B. Accumulated Response of EX to TX

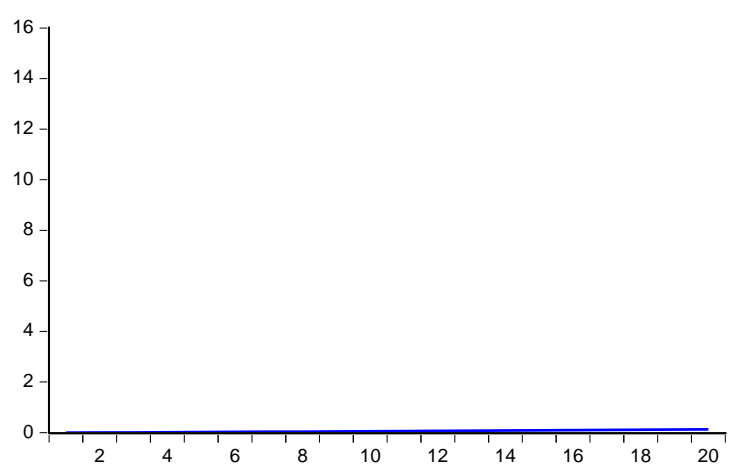


Panel C. Accumulated Response of TSE2 to EX

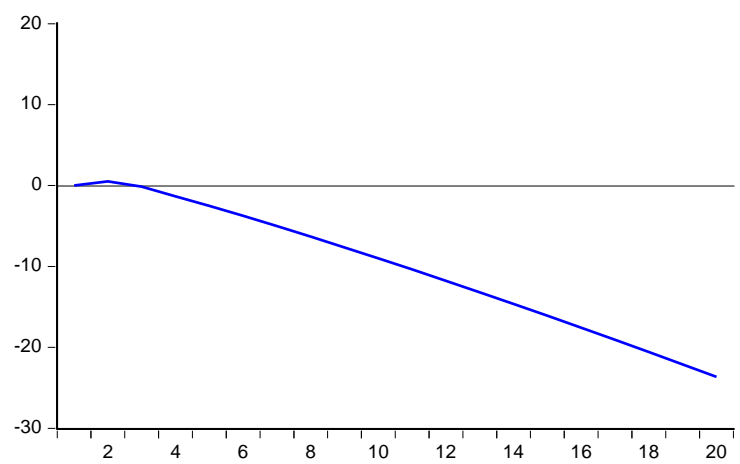

Panel E. Accumulated Response of TL70 to EX

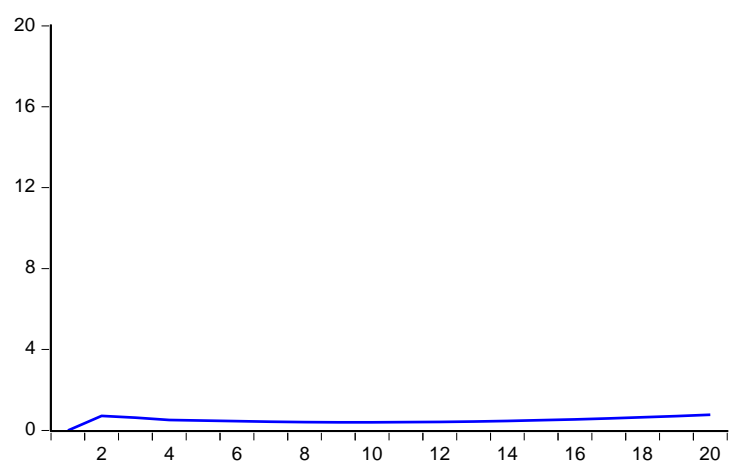

Panel G. Accumulated Response of TSM to EX

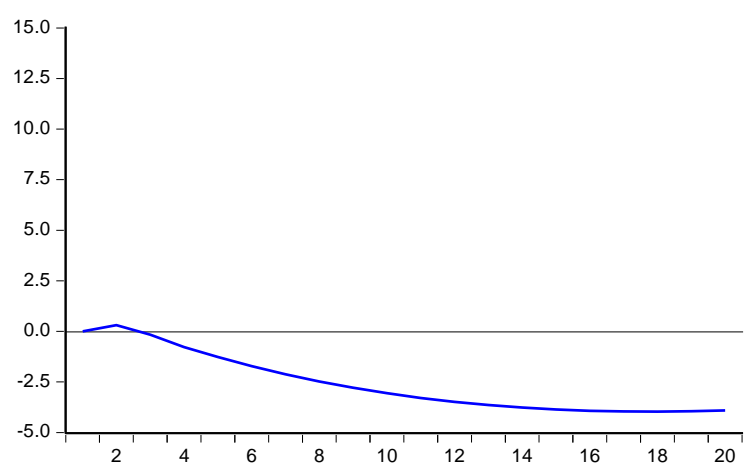

Panel D. Accumulated Response of EX to TSE2

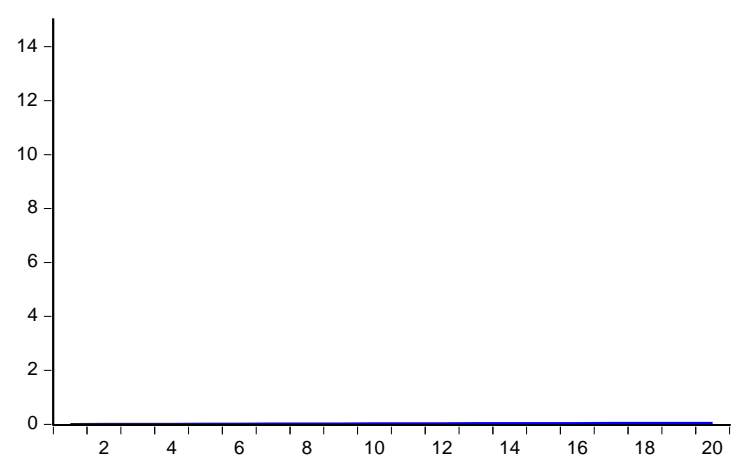

Panel F. Accumulated Response of EX to TL70

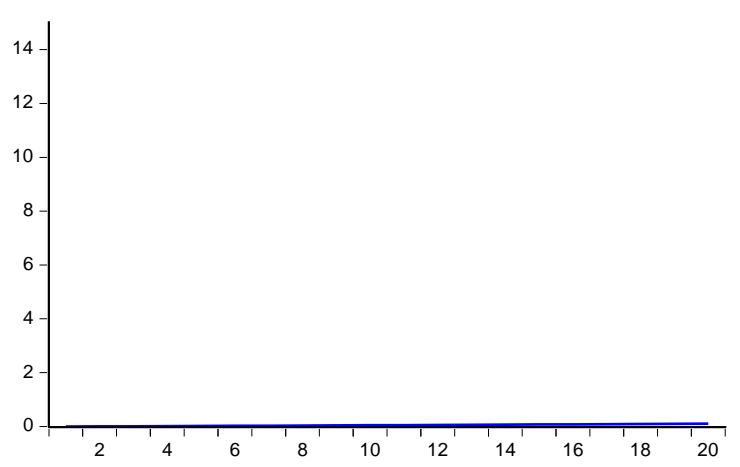

Panel H. Accumulated Response of EX to TSM

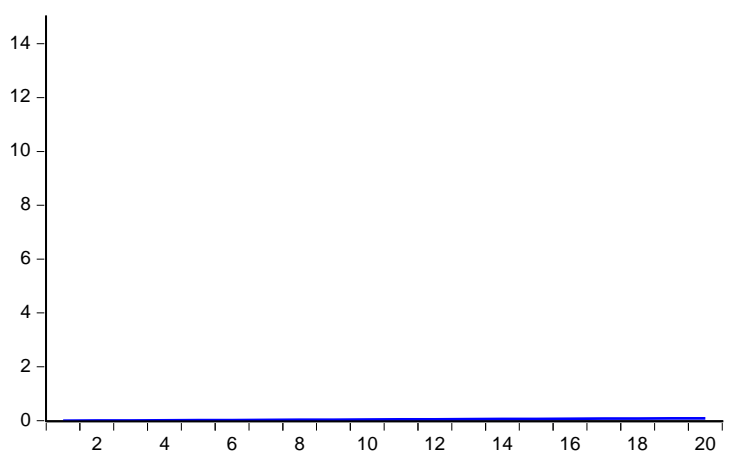

Figure 2. Impulse responses for the period from April 1, 2010 to November 13, 2012

Panel A. Accumulated Response of TX to EX

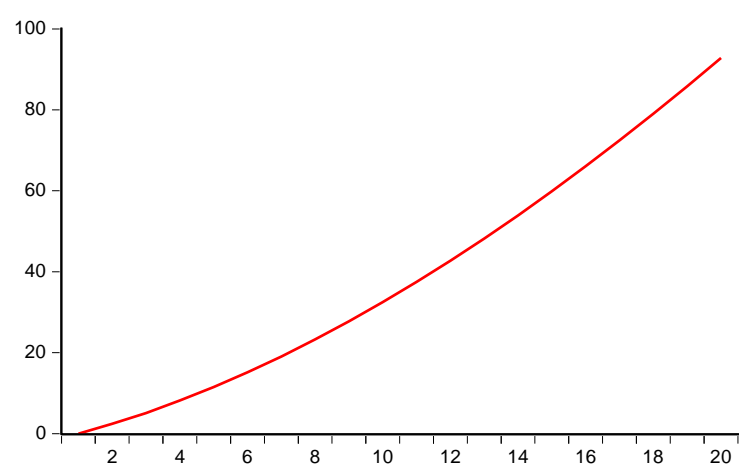

Panel B. Accumulated Response of EX to TX

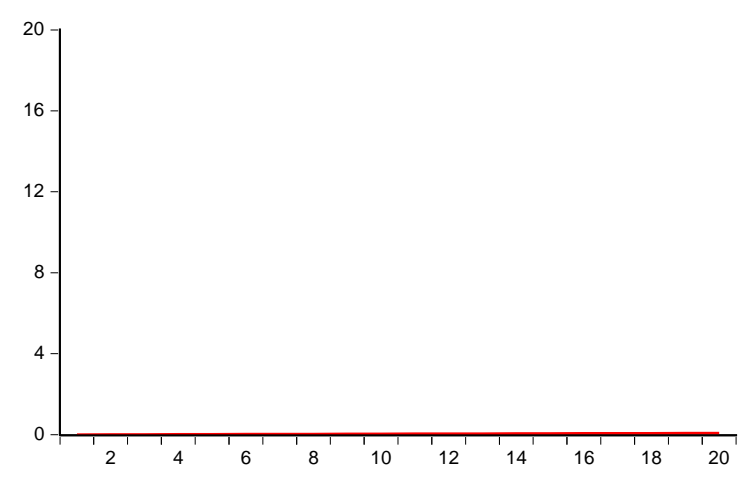


Panel C. Accumulated Response of TSE2 to EX

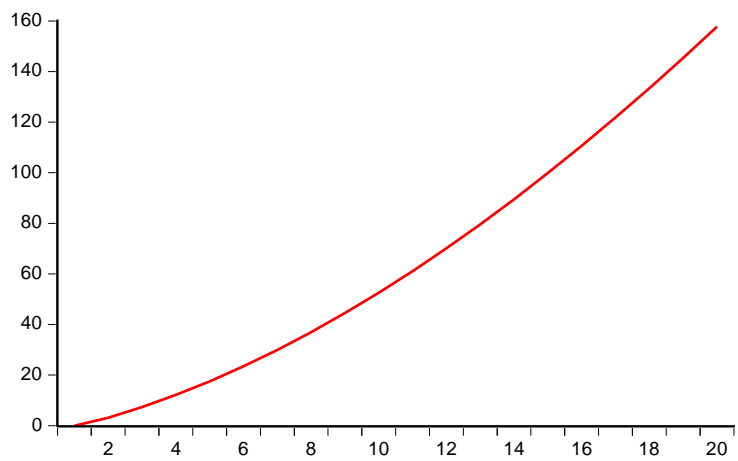

Panel E. Accumulated Response of TL70 to EX

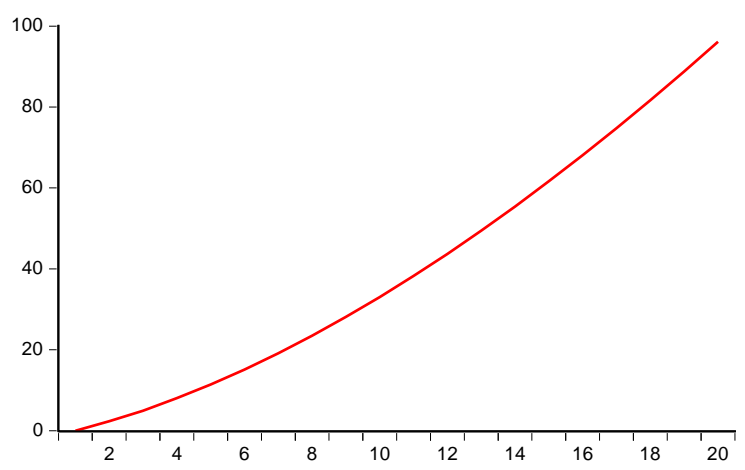

Panel G. Accumulated Response of TSM to EX

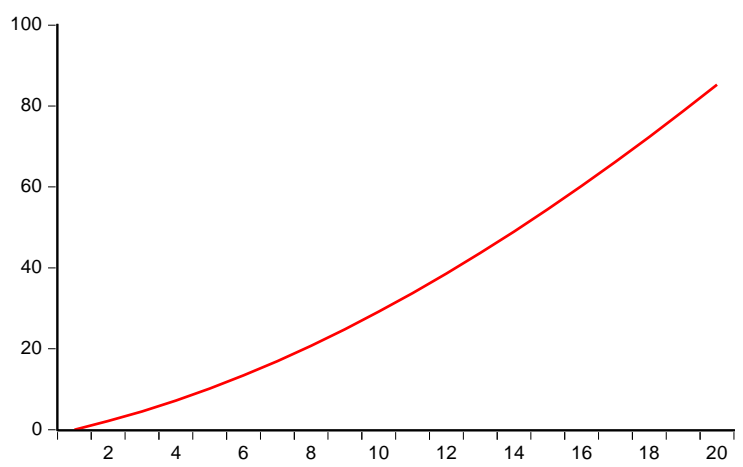

Panel D. Accumulated Response of EX to TSE2

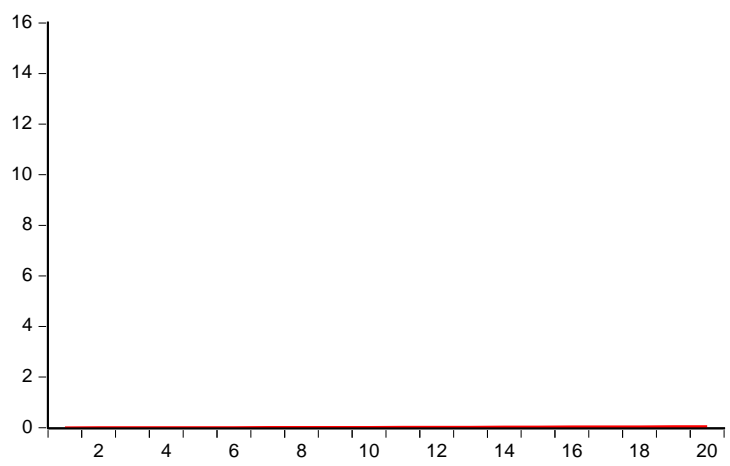

Panel F. Accumulated Response of EX to TL70

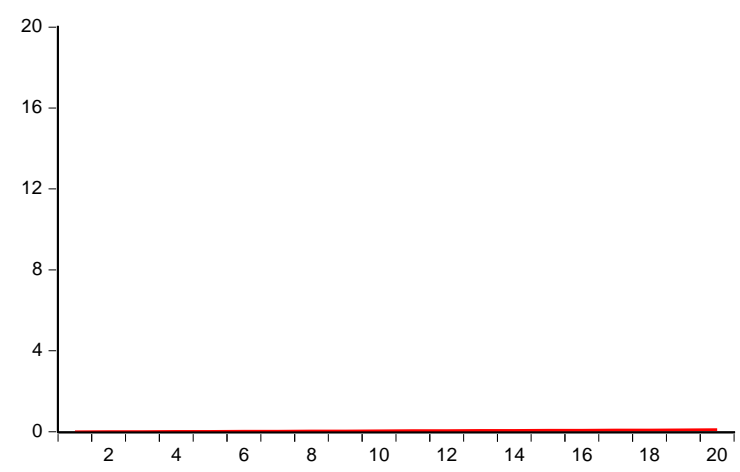

Panel H. Accumulated Response of EX to TSM

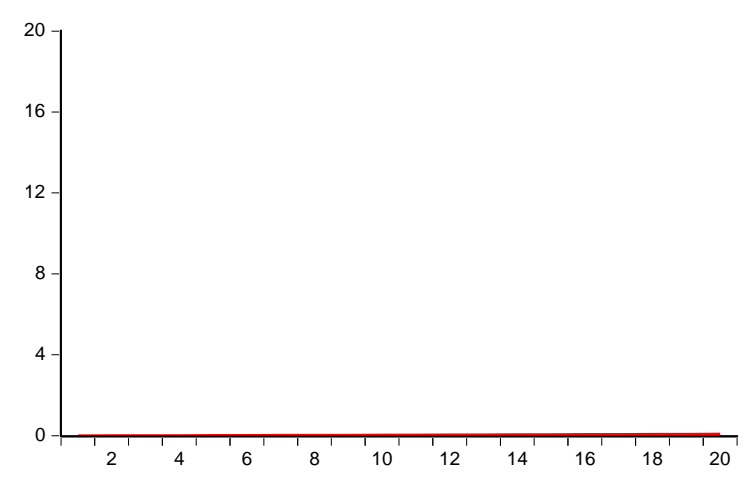

Figure 3. Impulse responses for the period from December 26, 2012 to August 18, 2015

\section{Empirical Results}

Regarding our empirical results, first, Figure 1 displays the time-varying correlation coefficients between the yen-US dollar exchange rate changes and stock returns, which are computed by taking 20-days windows. In all panels of Figure 1, the correlations for our first sample period are on the left side while the correlations for our second sample period are on the right side. In Figure 1, all panels indicate that, in the recent years, all our four Japanese stock return variables, TXLR, TSE2LR, TL70LR, and TSMLR exhibit much higher contemporaneous correlations with the changes in the yen-dollar exchange rate.

Next, Table 2 shows the estimation results as to our four kinds of bivariate Bayesian VAR models. In Table 2, Panel A displays the results for our first sample period and Panel B shows the results for our second sample period. In Panel B of this table, it is shown that in our second sample period, the first lag variables of EX are statistically significant in explaining the four Japanese stock index prices, TX, TSE2, TL70, and TSM. In addition, Panel B of Table 2 also shows that the first lag variables of TX, TL70, and TSM are statistically significant in explaining the 
movements of the exchange rate although all coefficients of the stock price lag variables are very small. Contrary to this, the estimation results of our VAR models displayed in Panel A of Table 2 indicate that there is little mutual effect between the yen-dollar exchange rate and the four kinds of Japanese stock index prices in our first sample period.

Based on the above results, for examining the degrees of the effects between stock prices and the exchange rate in our two sample periods, the mutual impulse response functions of EX, TX, TSE2, TL70, and TSM are depicted in Figures 2 and 3. Figure 2 exhibits their mutual responses for our first sample period and Figure 3 shows those for our second sample period, respectively. Figure 2 suggests that in our first sample period, EX shows little response to the shock in stock prices and stock prices do not exhibit clear response to the shock in EX. On the other hand, Figure 3 demonstrates that, in our second sample period, EX again shows almost no response to the shock in equity prices; however, all four Japanese stock index prices of TX, TSE2, TL70, and TSM exhibit much strong positive responses to the shock in EX. Therefore, our results of impulse response analyses suggest that, in the recent years, the past yen-dollar exchange rate series much more strongly affect Japanese stock prices whilst past Japanese stock prices do not affect the yen-dollar exchange rate changes.

\section{Conclusions}

This paper investigated the recent effects between the yen-dollar exchange rate changes and Japanese stock prices. Applying bivariate Bayesian VAR models, we obtained several interesting findings. First, (1) our analyses using Bayesian VAR models found that, in our recent sample period, the first lag variables of the yen-dollar exchange rate were statistically significant in explaining the four stock index prices: the TOPIX, TSE Second Section stock index, TOPIX Large 70 stock index, and TOPIX Small stock index in Japan.

Second, (2) the results of our impulse response analyses indicated that the yen-dollar exchange rate little responded to the shock in the equity prices in Japan; however, all stock prices of the TOPIX, TSE Second Section stock index, TOPIX Large 70 stock index, and TOPIX Small stock index positively responded to the shock in the exchange rate rather strongly in our recent sample period. Thus, our results of impulse response analyses clearly suggested that, in the recent years, the past yen-dollar exchange rate evolution much more strongly affected Japanese stock prices. Moreover, (3) our analyses of the time-varying correlation coefficients between the yen-dollar exchange rate and Japanese stock index returns revealed that, recently, contemporaneous correlations between the exchange rate and stock returns in Japan much increased.

Although it is not sure whether these strong contemporaneous linkages between them continue long in the future; however, under these recent economic and financial circumstances, it is considered that, for example, the clear exit announcements and decisive actions related to the current US monetary policy shall further affect the yen-dollar exchange rate and Japanese stock prices simultaneously. Trends of the Chinese economy and financial markets may also strongly influence the Japanese financial markets. Thus when there are such events or shocks to the economy, the volatile financial markets in Japan shall be expected and in such cases, behaviors of foreign investors, which much increasingly affect the Japanese financial markets in the recent years, may also play further important role in determining the movements of the Japanese financial markets.

Furthermore, we note that the evidence derived from our empirical examinations in this paper is rather clear and hence further investigations by employing other methodology and incorporating other contexts, for instance, are our future tasks.

\section{Acknowledgements}

I am particularly grateful to the kind repeated invitation from the journal to write to this journal. I also appreciate the Japan Society for the Promotion of Science for their generous financial assistance to this research. I further thank Susan Sun and Kitty Hua for the kindness to this paper. Finally, I deeply thank all the Editors of this Journal for the kindness to my paper.

\section{References}

Abouwafia, H. E., \& Chambers, M. J. (2015). Monetary policy, exchange rates and stock prices in the Middle East region. International Review of Financial Analysis, 37, 14-28. http://dx.doi.org/10.1016/j.irfa.2014.11.001

Andrieş, A. M., Ihnatov, I., \& Tiwari, A. K. (2014). Analyzing time-frequency relationship between interest rate, stock price and exchange rate through continuous wavelet. Economic Modelling, 41, 227-238. http://dx.doi.org/10.1016/j.econmod.2014.05.013

Branson, W. H. (1983). Macroeconomic determinants of real exchange rates. In: R.J. Herring, (Ed.) Managing 
foreign exchange risk. Cambridge: Cambridge University Press.

Ca' Zorzi, M., Kocięcki, A., \& Rubaszek, M. (2015). Bayesian forecasting of real exchange rates with a Dornbusch prior. Economic Modelling, 46, 53-60. http://dx.doi.org/10.1016/j.econmod.2014.10.060

Ciner, C., Gurdgiev, C., \& Lucey, B, M. (2013). Hedges and safe havens: An examination of stocks, bonds, gold, oil and exchange rates. International Review of Financial Analysis, 29, 202-211. http://dx.doi.org/10.1016/j.irfa.2012.12.001

Frankel, J. A. (1983). Monetary and portfolio-balance models of exchange rate determination. In: J.S. Bhandari, B.H. Putnam, (Eds.) Economic interdependence and flexible exchange rates. Cambridge: MIT Press.

Gelman, M., Jochem, A., Reitz, S., \& Taylor, M. P. (2015). Real financial market exchange rates and capital flows. Journal of International Money and Finance, 54, 50-69. http://dx.doi.org/10.1016/j.jimonfin.2015.02.004

Ho, L. C., \& Huang, C. H. (2015). The nonlinear relationships between stock indexes and exchange rates. Japan and the World Economy, 33, 20-27. http://dx.doi.org/10.1016/j.japwor.2015.02.002

Liang, C. C., Lin, J. B., \& Hsu, H. C. (2013). Reexamining the relationships between stock prices and exchange rates in ASEAN-5 using panel Granger causality approach. Economic Modelling, 32, 560-563. http://dx.doi.org/10.1016/j.econmod.2013.03.001

Moore, T., \& Wang, P. (2014). Dynamic linkage between real exchange rates and stock prices: Evidence from developed and emerging Asian markets. International Review of Economics and Finance, 29, 1-11. http://dx.doi.org/10.1016/j.iref.2013.02.004

Petrevski, G., Exterkate, P., Tevdovski, D., \& Bogoev, J. (2015). The transmission of foreign shocks to South Eastern European economies: A Bayesian VAR approach. Economic Systems, Forthcoming. http://dx.doi.org/10.1016/j.ecosys.2015.04.003

Vithessonthi, C., \& Tongurai, J. (2013). Unremunerated reserve requirements, exchange rate volatility, and firm value. Journal of International Financial Markets, Institutions \& Money, 23, 358-378. http://dx.doi.org/10.1016/j.intfin.2012.10.004 